\title{
On a simple and accurate quantum correction for Monte Carlo simulation
}

\section{Journal Article}

\section{Author(s):}

Bufler, F.M.; Hude, R.; Erlebach, A.

Publication date:

2006-12

Permanent link:

https://doi.org/10.3929/ethz-b-000040638

Rights / license:

In Copyright - Non-Commercial Use Permitted

Originally published in:

Journal of Computational Electronics 5(4), https://doi.org/10.1007/s10825-006-0038-1 


\title{
On a simple and accurate quantum correction for Monte Carlo simulation
}

\author{
F. M. Bufler · R. Hudé · A. Erlebach
}

Published online: 9 December 2006

(C) Springer Science + Business Media, LLC 2007

\begin{abstract}
We investigate a quantum-correction method for Monte Carlo device simulation. The method consists of reproducing quantum mechanical density-gradient simulation by classical drift-diffusion simulation with modified effective oxide thickness and work function and using these modifications subsequently in Monte Carlo simulation. This approach is found to be highly accurate and can be used fully automatically in a technology computer-aided design (TCAD) workbench project. As an example, the methodology is applied to the Monte Carlo simulation of the on-current scaling in $p$-and $n$-type MOSFETs corresponding to a $65 \mathrm{~nm}$ node technology. In particular, it turns out that considering only the total threshold voltage shift still involves a significant difference to a Monte Carlo simulation based on the combined correction of oxide thickness and work function. Ultimately, this quantum correction permits to consider surface scattering as a combination of specular and diffusive scattering where the conservation of energy and parallel wave vector in the specular part takes stress-induced band structure modifications and hence the corresponding surface mobility changes on a physical basis into account.
\end{abstract}

Keywords Monte Carlo device simulation · Quantum correction $\cdot$ TCAD

\author{
F. M. Bufler $(\bowtie)$ \\ Institut für Integrierte Systeme, ETH Zürich, Gloriastrasse 35, \\ CH-8092 Zürich, Switzerland \\ e-mail: bufler@iis.ee.ethz.ch \\ R. Hudé · F. M. Bufler · A. Erlebach \\ Synopsys Schweiz GmbH, Affolternstrasse 52, CH-8050 Zürich, \\ Switzerland \\ e-mail: hude@synopsys.com \\ A. Erlebach \\ e-mail: erlebach@synopsys.com
}

\section{Introduction}

As MOSFETs are scaled into the nanometer regime, fullband Monte Carlo simulation becomes mandatory to estimate quasi-ballistic transport without prior calibration. At the same time, also the importance of quantum effects is increased. This raises the question of how quantum corrections can be incorporated into Monte Carlo simulation and several approaches have been proposed in the literature: (i) quantization perpendicular to the silicon/oxide interface is considered via the solution of the Schrödinger equation and transport is performed in the resulting subbands (e.g. [1-3]), (ii) an effective quantum potential is used (e.g. [4, 5]) or (iii) a quantum-corrected position-dependent band structure is employed (e.g. [6]). While all these approaches feature an electron density profile, which tends to vanish at the interface, the corresponding formulation of surface roughness scattering and/or the application to realistic MOSFETs is problematic. First, using directly or indirectly the Schrödinger equation (e.g. to determine the parameters of an effective potential) involves an arbitrary choice of the transition point between bulk and quantization region both in space and in energy, and requires for computational reasons analytical band structure descriptions. As quantization already leads in case (i) to a valley repopulation, this approach fails to reproduce the experimental strain-induced electron mobility enhancements [3]. In cases (ii) and (iii), the model for surface roughness scattering based on the surface roughness root mean square and correlation length [4] still inconsistently involves in the full-band versions $[5,6]$ an effective mass and requires straindependent calibration to measurements [5].

In contrast, a combination of specular and diffusive scattering at the interface [7] explicitly involves the straindependence via the conservation of energy and parallelmomentum in the specular part $(85 \%$ in our Monte Carlo 
simulator SPARTA). In fact, this model leads for holes under biaxial tensile strain to a much lower enhancement for the surface mobility than for the bulk mobility [8] in agreement with surface mobility measurements [9]. However, this approach necessitates a classical density profile allowing electrons to hit the surface (increasing the diffusive part for a quantum density profile would eliminate the strain dependence for this mechanism, if feasible at all). A possibility to combine this surface scattering model with quantum effects is to use a modification of oxide thickness and work function as obtained from quantum mechanics [10]. In [10], this was achieved by analytical approximations for the threshold voltage shift in weak inversion and for the effective oxide thickness increase in strong inversion based on a parabolic electron band structure. It is the aim of this paper to generalize this method to a completely numerical approach, which can be routinely applied in a TCAD environment, to demonstrate its accuracy and to apply it to process-simulated $p$ - and $n$-MOSFETs of a $65 \mathrm{~nm}$ technology.

\section{Approach and discussion}

Figures 1 and 2 show the source-side of the channel in a 45 $\mathrm{nm} n$-MOSFET and the threshold voltage roll-off curves of $n$ and $p$-MOSFET as obtained by density-gradient (DG) simulations, respectively. Our quantum-correction method consists of reproducing the DG simulation below and around the threshold by a classical drift-diffusion (DD) simulation with modified work function and oxide thickness and to employ these modified values subsequently in full-band Monte Carlo simulation. First, the increase of the effective oxide thickness in the on-state is computed according to $\Delta t_{\mathrm{ox}}=\left(X_{\mathrm{qm}}-X_{\mathrm{cl}}\right)$ $\epsilon_{\mathrm{ox}} / \epsilon_{\mathrm{Si}}$ where the quantum mechanical and classical charge centroids are calculated in the source-side of the channel along the line depicted in Fig. 1. In a 2nd classical DD simu-

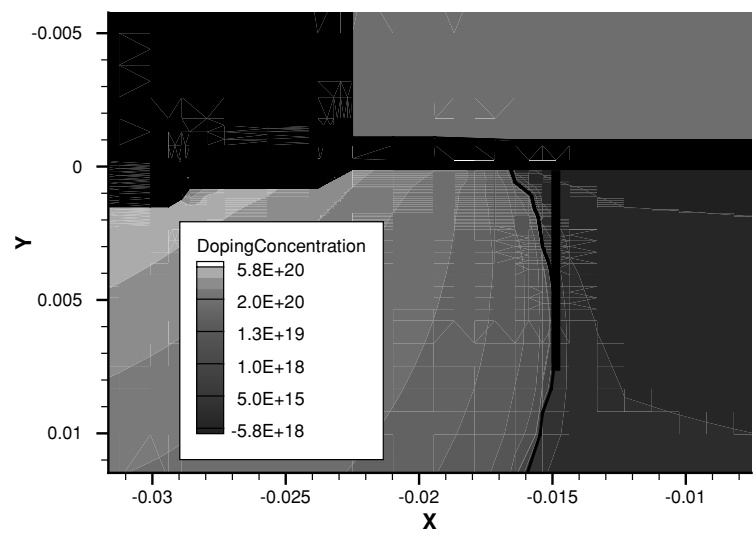

Fig. 1 Cross section of the source side of the channel in a $45 \mathrm{~nm}$ $n$ MOSFET. The bold vertical bar shows the position where the charge centroids are calculated from the electron density profiles

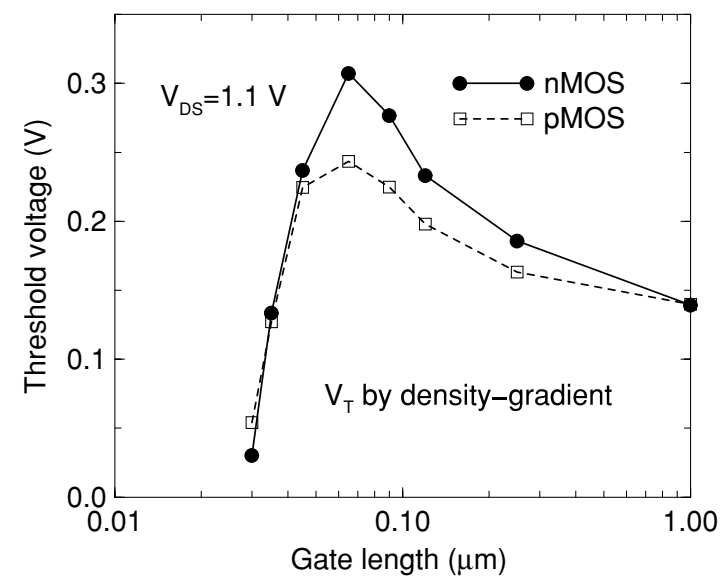

Fig. 2 Saturation threshold voltage roll-off curves of $n$ MOSFET and $p$ MOSFET for a $65 \mathrm{~nm}$ node technology according to density-gradient simulations

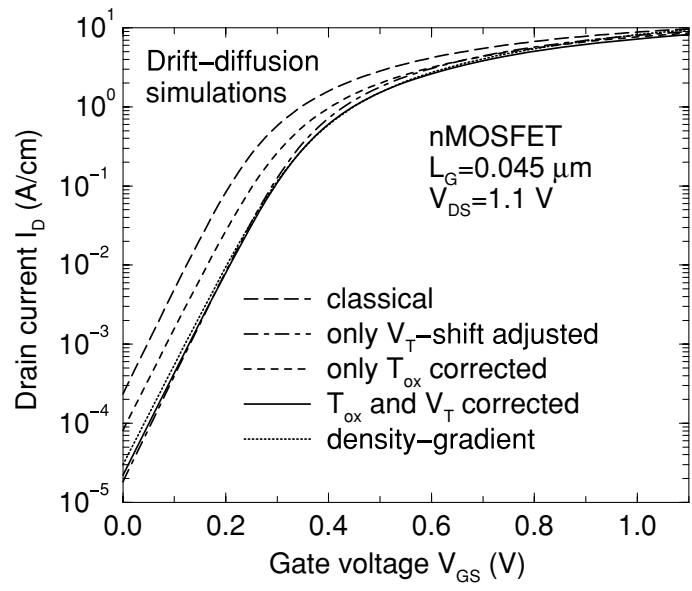

Fig. 3 Logarithmic plot of the transfer characteristics of the $45 \mathrm{~nm}$ $n$ MOSFET showing the results of different quantum corrections employed in drift-diffusion simulations

lation using this $\Delta t_{\mathrm{ox}}$, the remaining threshold voltage shift to the DG simulation is extracted and considered as a modified work function in the 3rd DD simulation as well as in the Monte Carlo simulation.

In Figs. 3 and 4, the logarithmic and linear plots of the transfer characteristics are shown for the $45 \mathrm{~nm} n$-MOSFET according to classical DD simulation, DD simulation where only the threshold voltage shift is considered (this shift is, of course, larger than the remaining shift when already taking $\Delta t_{\mathrm{ox}}$ into account), DD simulation where only the increased $t_{\mathrm{ox}}$ is considered, the final DD simulation with modified $t_{\mathrm{ox}}$ and corrected remaining threshold voltage shift, and DG simulation. In the subthreshold regime, i.e. in weak inversion, changing only $t_{\mathrm{ox}}$ is not sufficient while considering only the threshold voltage shift matches the DG result, whereas both modifications are similar somewhat above threshold, but still significantly away from density-gradient. Only the combined correction reproduces density-gradient up to far above threshold and also matches the quantum mechanical 


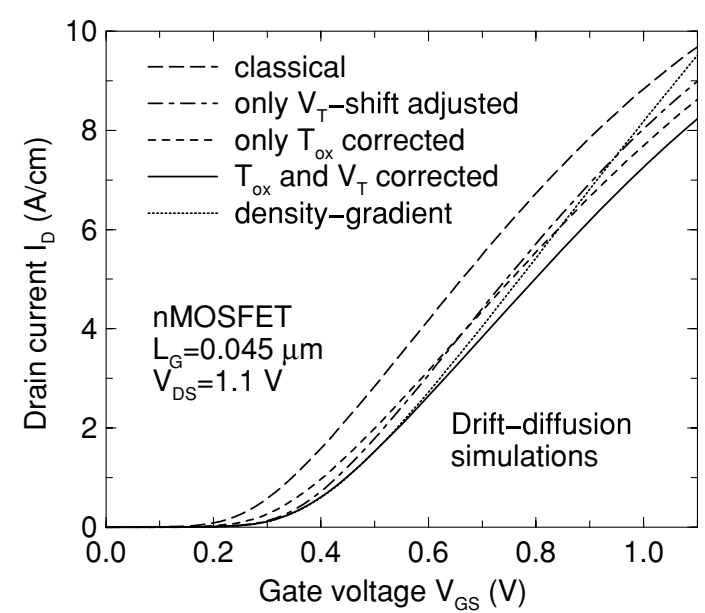

Fig. 4 Linear plot of the transfer characteristics of the $45 \mathrm{~nm} n$ MOSFET showing the results of different quantum corrections employed in driftdiffusion simulations

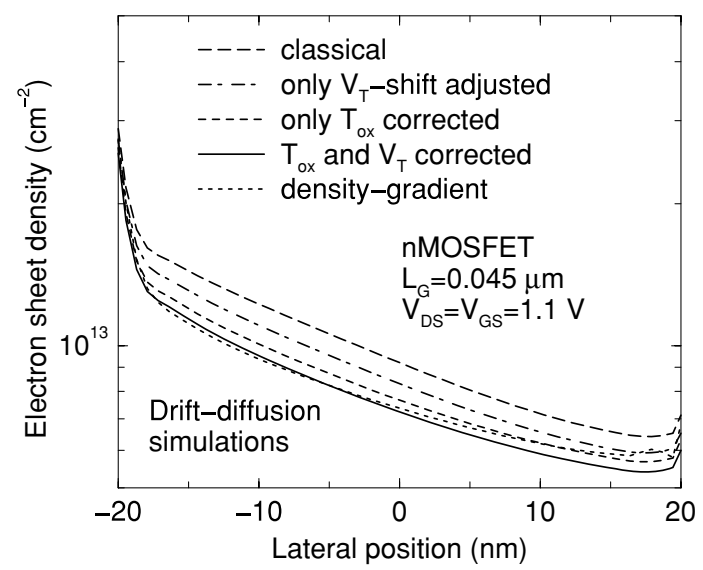

Fig. 5 Electron sheet densities along the channel in the $45 \mathrm{~nm}$ $n$ MOSFET as present for different quantum corrections within driftdiffusion simulations

electron sheet density in the source-side of the channel (see Fig. 5).Finally note that the current at high gate voltage is determined by the surface mobility model which in principle should have been recalibrated to the universal mobility curve for the DG simulation (in fact, this would lead to a complete agreement of the 2 curves).

In Fig. 6, the results of the different corrections when used in Monte Carlo simulation are shown for the oncurrent scaling of $n$ - and $p$-MOSFET. Of course, the $t_{\mathrm{ox}}$ correction has the strongest effect because the on-state corresponds to strong inversion, but the additional threshold voltage shift has a noticeable effect. In particular, considering only the total threshold shift still involves a significant difference to the final result based upon the combined correction.

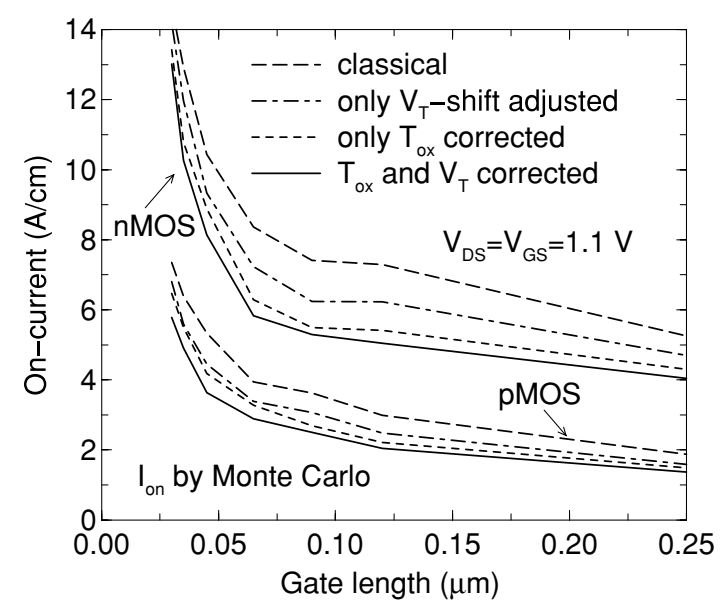

Fig. 6 On-current scaling according to full-band Monte Carlo simulations of $n$ MOSFETs and $p$ MOSFETs using different quantum corrections

\section{Conclusions}

We have presented a quantum-correction scheme for fullband Monte Carlo simulation which considers the quantum mechanical increase of effective oxide thickness and threshold voltage. In a TCAD environment, this method can be used fully automatical in a workbench project and allows to use consistently diffusive and specular surface roughness scattering and the corresponding stress-dependence.

\section{References}

1. Hao, C. et al.: Monte Carlo study of two-dimensional electron gas transport in Si-MOS devices. Solid-State Electron. 28, 733 (1985)

2. Jungemann, C. et al.: Simulation of linear and nonlinear electron transport in homogeneous silicon inversion layers. Solid-State Electron. 36, 1529 (1993)

3. Fischetti, M.V. et al.: On the enhanced electron mobility in strainedsilicon inversion layers. J. Appl. Phys. 92, 7320 (2002)

4. Ramey, S.M., Ferry, D.K.: Implementation of surface roughness scattering in Monte Carlo modeling of thin SOI MOSFETs using the effective potential. IEEE Trans. Nanotechnol. 2, 110 (2003)

5. Pham, A.T. et al.: A semiempirical surface scattering model for quantum corrected Monte Carlo simulation of strained SinMOSFETs. In: Proceedings of the ESSDERC, pp. 293-296 (2005)

6. Fan, X.-F. et al.: MC simulation of strained-Si MOSFET with fullband structure and quantum correction. IEEE Trans. Electron Devices 51, 962 (2004)

7. Sangiorgi, E., Pinto, M.R.: A semi-empirical model of surface scattering for Monte Carlo simulation of silicon $n$-MOSFET's. IEEE Trans. Electron Devices 39, 356 (1992)

8. Keith, S. et al.: Full band Monte Carlo device simulation of 0.1$0.5 \mu \mathrm{m}$ strained-Si $p$-MOSFETs. In: Proceedings of the ESSDERC, pp. 312-315 (1998)

9. Currie, M.T. et al.: Carrier mobilities and process stability of strained Si $n$ - and $p$-MOSFETs on SiGe virtual substrates. J. Vac. Sci. Technol. B 19, 2268 (2001)

10. Hudé, R. et al.: A simple approach to account for the impact of quantum confinement on the charge in semiclassical Monte Carlo simulations of bulk nMOSFETs. In: Proceedings of the ULIS, pp. 159-162 (2005) 